\title{
Deformable Microsystem for In Situ Cure Degree Monitoring of GFRP (Glass Fiber Reinforced Plastic)
}

\author{
Yang Yang ${ }^{1,2,3}$, Gabriele Chiesura ${ }^{2}$, Thomas Vervust ${ }^{1,2,3}$, Frederick Bossuyt ${ }^{1,2,3}$, \\ Geert Luyckx ${ }^{2}$, Markus Kaufmann ${ }^{4}$, Joris Degrieck ${ }^{2}$, and Jan Vanfleteren ${ }^{1,2,3}$ \\ ${ }^{1}$ Center for Microsystems Technology, Ghent, Belgium \\ ${ }^{2}$ Ghent University, Ghent, Belgium. \\ ${ }^{3}$ IMEC, Leuven, Belgium. \\ ${ }^{4}$ Sirris Leuven-Gent Composites Application Lab, Leuven, Belgium.
}

\begin{abstract}
Fibre Reinforced Polymer (FRP) is becoming a valid alternative to many traditional heavy metal industries because of its high specific stiffness over the more classical construction metals. Recent trend of more complex geometry of composites is causing increasing difficulty in composite manufacturing. A method to optimize the manufacturing process is thus imposed to ensure and improve the quality of manufactured parts. Because of the irregular 3D shapes of the composites, traditional flat sensor system is becoming unfavorable and nonpractical for monitoring purpose. In this work, the current development status of a deformable microsystem for in situ cure degree monitoring of a glass fibre reinforced plastic is presented. To accommodate the non-flat shape of the composites, the proposal is to interconnect nondeformable functional island, which contains the capacitive sensor for cure degree monitoring, with meander-shaped deformable interconnections. The developed sensor system is able to withstand the manufacturing process where change of pressure and internal strain, thus force exerted on the sensor system, is involved.
\end{abstract}

\section{INTRODUCTION}

Fibre Reinforced Polymer (FRP) is becoming a valid alternative to many traditional heavy metal industries because of its high specific stiffness over the more classical construction metals. Due to increasing difficulties in manufacturing of FRP composites caused by a trend of more complex, very often 3D, composite geometry, a method to optimize the manufacturing process of FRP is imposed such that different steps (e.g. injection of resin, curing and postcuring) can be better understood to have good quality of the produced part while keeping manufacturing costs minimum. Because of the irregular 3D shapes of the expected composite parts, traditional flat sensor system turns out to be outdated or increasingly difficult to be used in combination with the novel manufacturing process. In this study, the current development status of a stretchable and ultimately deformable microsystem for in situ cure degree monitoring of a glass fibre reinforced plastic fabricated by a resin infusion process is presented. Interdigital type of capacitive sensor is designed, fabricated and embedded for monitoring different manufacturing steps, especially the cure degree of composites. Curing phase is certainly an important stage during manufacturing of a composite component, during which the polymer constitute of composites cross-links, results in the consolidation of the whole composite structure. By controlling the curing phase one can obtain a superior quality component and, therefore, will increase the reliability and optimize the design of the part, leading to a decrease of the lifecycle cost. To accommodate the non-flat shape of the composites, the idea is to combine 
flexible, yet non-deformable functional island containing the sensor with the deformable metallic interconnects. The circuit comprises a flexible island, where the island holds and contains a single capacitive sensor as the cure degree monitoring unit. The developed sensor system is flexible and deformable, and is able to withstand the manufacturing process where large and sudden change of air pressure, thus force exerted on the sensor system, is involved.

\section{EXPERIMENT}

\section{Deformable Microsystem}

Future sensor system should be deformable to arbitrary 3D shapes to accommodate the complex geometry and novel manufacturing process of the composites, this means at least parts of the sensor system should survive the deformation. On the other hand, the sensor itself should retain a reasonable performance and a reliable measurement during and after the deformation. The idea is thus to interconnect flexible, yet non-deformable functional islands containing the sensor unit or other intelligence with the deformable interconnections [1]. Note that deformable, instead of stretchable, is used as the terminology here, to differentiate dynamic multi-cycle stretchability from semi-static deformability which happens mostly during a composite manufacturing process. In this study, we investigated a simple sensor system with one single island interconnected to the external sensor readout via meander-shaped interconnections as shown in Fig. 1. Gradual transitions in width of the copper and corresponding supporting polyimide reduce the local strain in the copper during the deforming [2]. To demonstrate the deformability of this sensor system, a vacuum assisted resin infusion process for fabricating the FRP is selected and will be elaborated later.

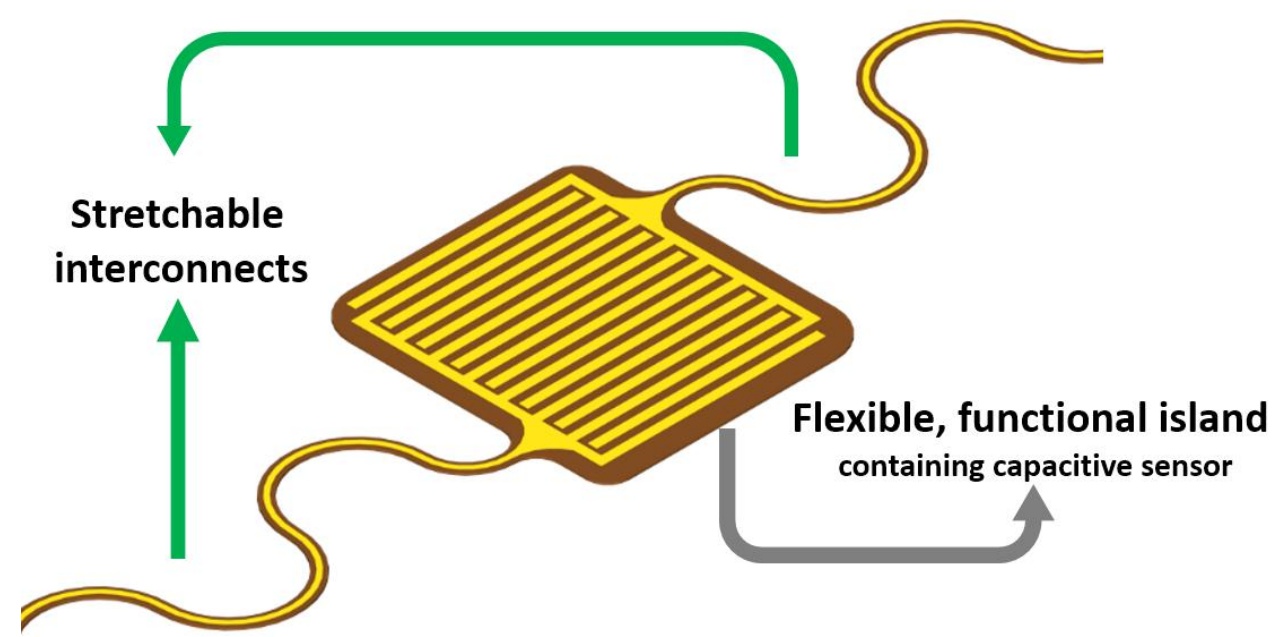

Fig. 1 Capacitive sensor in combination with deformable interconnections

Fig. 2 (a) shows the interdigital type of capacitive sensor used in this study fabricated by flexible circuit board technology. The sensor fabrication starts from patterning a polyimidecopper laminate into interdigital structure by lithography and spray etching process, afterwards a thin coating of Organic Solderability Preservative (typical thickness 0.15-0.3 micron) is applied on top of the copper electrode to protect it from oxidation. The resulting sensor is $68 \mu \mathrm{m}$ in thickness, and $15 \mathrm{~mm} \times 12 \mathrm{~mm}$ in size. Fig. 2(b) shows the schematic drawing of the capacitive sensor: w stands for the width of each 'finger', g stands for the gap (spacing) between fingers, 
and L stands for the finger length. When an electric potential is applied between the anode and cathode of the sensor, an electric field is created between the fingers, and extended to the space on both sides of the finger structure to a certain distance. This depth (distance), that the majority of the electric field is able to reach, is termed the penetration depth of the sensor, and determined by the spatial wavelength $(w+g)$ of the structure [3]. The sensing depth calculated for our current design is approximately $100 \mu \mathrm{m}$, as such the sensor response is only affected by the change at the vicinity of the sensor surface. Polyimide is used as the substrate material as it exhibits a stable dielectric constant over a wide temperature range. A corresponding sensor capacitance deviation in air of $0.02 \mathrm{pF} /{ }^{\circ} \mathrm{C}$ in the temperature range $20-80{ }^{\circ} \mathrm{C}$ is found for our current design, which is almost negligible compared to the change caused by polymer curing.

(a)

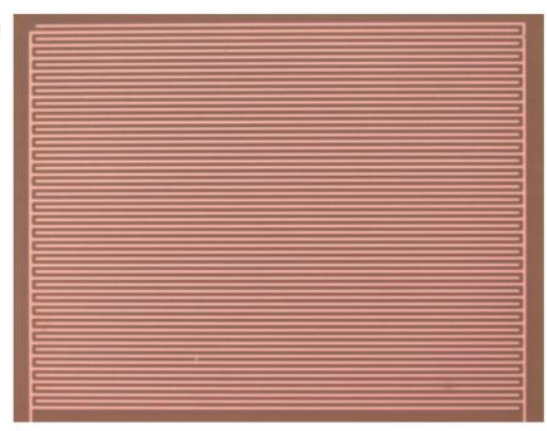

(b)

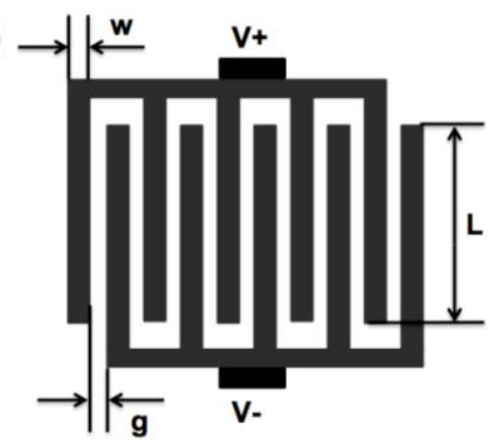

Fig. 2 (a) IDC geometry; (b) Fabricated capacitive sensor with $\mathrm{w}=\mathrm{g}=100 \mu \mathrm{m}, \mathrm{L}=15 \mathrm{~mm}$

\section{Vacuum Assisted Resin Infusion Process}

To demonstrate the deformability of this sensor system, a vacuum assisted resin infusion process for fabricating the FRP is selected. As we are still in the early phase of the development, a flat glass mould instead of a real 3D mould was selected for the proof of concept. During the fabrication process, change of pressure within the vacuum bagging due to the use of pump, as well as change of composite internal strain due to the curing of resin would happen; these together result in a force or source of deformation on the sensor system. Six layers of UDO ES 500 UD Glass Fibre reinforcements were laid up on top of a flat glass mould, a flexible interdigital and temperature sensor island in conjunction with meander-shaped deformable interconnects were embedded between glass fibres in the midmost layer. Additionally, there was one thermocouple outside the mould for ambient temperature measurement. The plate was sealed on the four edges with vacuum bag. The epoxy matrix system (from Momentive) in use is a twopart system consisting of the EPIKOTE MGS RIMR 135 resin and the EPIKURE MGS RIMH 137 hardener. $300 \mathrm{~g}$ of RIMR135 and 90g of RIMH137 were thoroughly mixed by a mechanical blender, degassed, and infused to the vacuum bagging through the resin inlet, the outlet was connected with a tube to the vacuum line, and once the resin reaches the outlet, both tubes were clamped to stop the resin flow and keep the bag under vacuum. The glass fibre reinforced composite was cured at room temperature for $24 \mathrm{~h}$, followed by a post cure in the oven at $80^{\circ} \mathrm{C}$ for $16 \mathrm{~h}$ as recommended by the datasheet. During the process, the possible deformation on the sensor system will be accommodated by the meander-shaped interconnects to maintain the electrical connection of the sensor system. On the other hand, due to a high tensile strength about $499 \mathrm{~N} / \mathrm{mm}^{2}$ of the polyimide substrate in conjunction with the flatness of the sensor island 
maintained by the vacuum, it can therefore be assumed that the sensor island retains its geometry during the resin infusion process to ensure a consistent sensor response. A self-made readout system comprising a microcontroller was designed and fabricated for the capacitance and temperature measurement. The acquisition time of each measurement is about $500 \mathrm{~ms}$.

\section{DISCUSSION}

The capacitance measurement for the resin cured at room temperature is shown in Fig 3. At the beginning of cure, a large increase in capacitance was identified at approximately minute 12 ( $0.2 \mathrm{~h}$ in Fig. 3a): indicating that the resin has reached the capacitive sensor, and the maximum value indicates the resin has completely covered the capacitive sensor. Zooming into the figure, we are able to see that it took around 2 minutes before the uprising curve reaches the maximum value. The large increase in capacitance is caused by the change of the dielectric layer above the sensor from air to resin as well as the ionic impurities in the resin. As the cure progresses, cross-linking between monomers or oligomers is continued and a network is expanded. The resin system becomes more viscous and the ionic diffusion gets restricted. The restricted ionic diffusion results in a decrease of conductivity. At around 12 hours into the cure (Fig. 3b), a relatively steep decrease in capacitance is observed indicating the transition of the resin from a gelly state into a glassy state, and is referred to gelation point or vitrification point [4]. As the resin becomes more viscous during the curing, the translational diffusion of ion and the rotational diffusion of dipole moment are not able to follow the excitation signal, and needs longer time for the reorientation and relaxation.

(a)

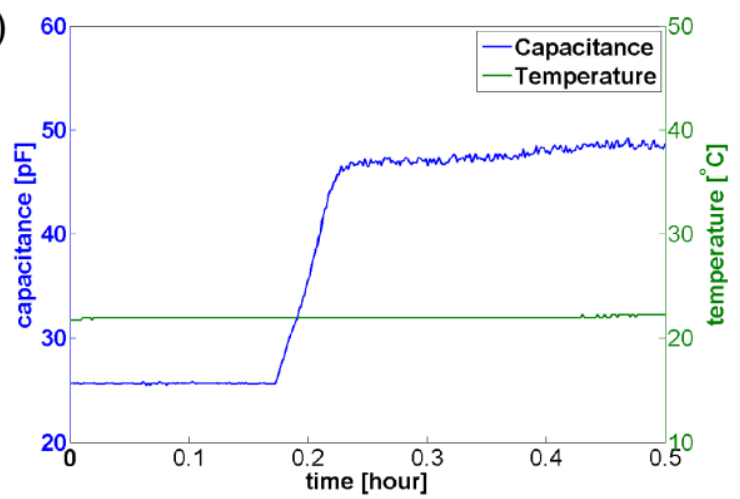

(b)

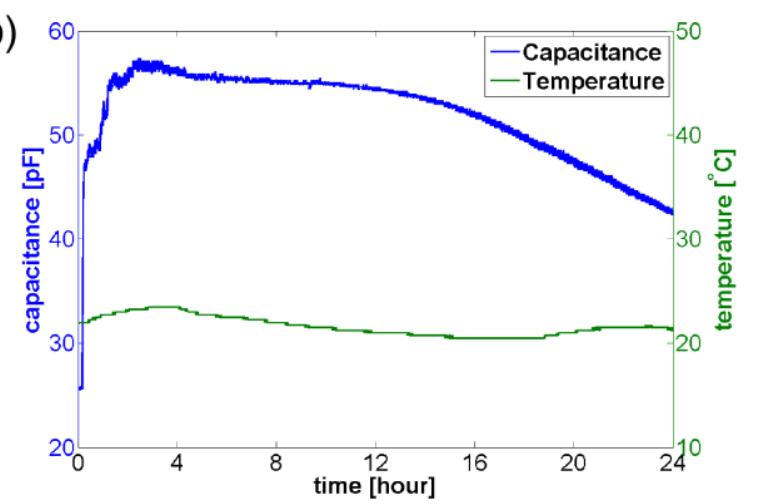

Fig. 3 Capacitance and temperature change of resin (a) for the first half hour (b) for the first $24 \mathrm{~h}$ during a vacuum assisted resin infusion process

During post-curing, the heating up of the oven leads to an increase in resin temperature as shown in Fig. 4. A large increase in capacitance is observed in the beginning of post curing, after reaching the maximum, a rapid decrease of capacitance was seen, and the slope became smaller. The overshoot of capacitance indicates further polymerization within the resin, when the temperature is raised above glass transition temperature $(\mathrm{Tg})$. After the peak, capacitance values decrease and saturate to nearly stable values since the oven is kept at $80^{\circ} \mathrm{C}$. After $16 \mathrm{~h}$, the oven is turned off, and the decrease in temperature causes the drop in capacitance, as the dielectric property is temperature dependent, but the change in absolute value is considered insignificant compared to the peak observe at the beginning of post-curing or change during the curing stage. As can be seen from the plot, the capacitance measurement gives information about resin cure that is mostly related to degree of cure rather than the influence of the ambient environment. 


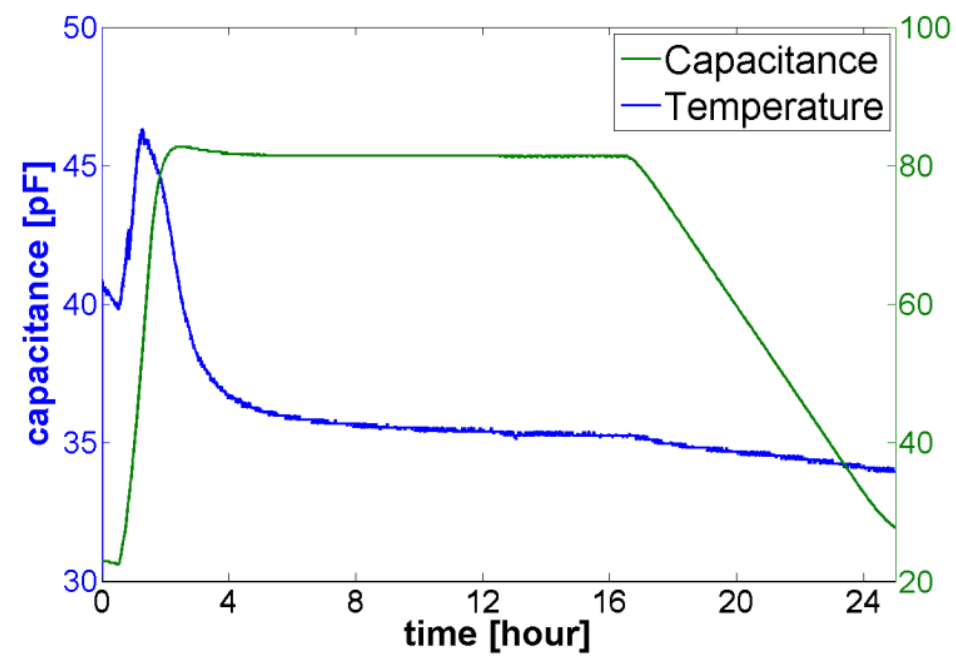

Fig. 4 Capacitance and temperature change of resin during the post-curing

\section{CONCLUSIONS}

Next generation of sensor system should be compatible with novel manufacturing process of composites where deformation of the sensor system to arbitrary 3D shapes takes place. In this study, we presented the current status of the development of a deformable microsystem for in situ cure degree monitoring of a glass fibre reinforced plastic fabricated by a resin infusion process. The developed sensor system combines flexible, yet non-deformable island containing the functional unit (capacitive sensor for cure degree monitoring in this case) with deformable meander-shaped interconnections. During the vacuum assisted resin infusion process, fabricated sensor system is shown to be stable throughout the fabrication process, and is able to withstand the change of pressure and resin internal strain, thus source of deformation exerted on the sensor system.

\section{ACKNOWLEDGMENTS}

The authors thank S. Dunphy, S. Van Put, and K. Dhaenens for fabricating the capacitive sensors, J. Windels for helping with hardware related issues. The research leading to these results has received funding from the Flemish Agency for Innovation by Science and Technology (IWT) - through the program for Strategic Basic Research (SBO) under grant agreement $\mathrm{n}^{\circ} 120024$ (Self Sensing Composites).

\section{REFERENCES}

1. A. V. Mamishev, K. Sundara-Rajan, Y. Fumin, D. Yanqing, M. Zahn, Interdigital sensors and transducers, Proceedings of the IEEE 92 (5) (2004) 808-845.

2. J. Vanfleteren, M. Gonzalez, F. Bossuyt, Y-Y Hsu, T. Vervust, I. De Wolf, M. Jablonski, Printed circuit board technology inspired stretchable circuits, MRS BULLETIN, 2012;37(3):254-60. 
3. F. Bossuyt, T. Vervust, J. Vanfleteren, Stretchable electronics technology for large area applications: fabrication and mechanical characterization. IEEE TRANSACTIONS ON COMPONENTS PACKAGING AND MANUFACTURING TECHNOLOGY. 2013;3(2):22935.

4. G. M. Maistros, C. B. Bucknall, Modeling the dielectric behavior of epoxy-resin blends during curing, Polymer Engineering and Science 34 (20) (1994) 1517-1528. 